\title{
Ischaemia of peripheral nerve and muscle
}

\author{
D. G. F. HARRIMAN \\ From the General Infirmary at Leeds
}

Both peripheral nerve and muscle are resistant to ischaemia and are capable of metabolic activity under anaerobic conditions. Despite this, there are many clinical syndromes in man associated with nerve ischaemia and ischaemic muscle disease is not uncommon, although a neglected subject for study. In the following account, the direct effects of ischaemia on nerve and muscle will be described, but the possible secondary effects on muscle of ischaemic neuropathy, namely, neurogenic atrophy and disuse atrophy (Adams, 1975), will be omitted. They need to be borne in mind, however, when examining muscle.

\section{Peripheral nerve}

\section{BLOOD SUPPLY}

A good account has been given by Sunderland (1945a, b, and c) based on his findings in man, and current views on vascular permeability are discussed by Low (1976). Regional arteries provide nutrient branches which enter the epineurium at intervals. Here the arteries and veins form a longitudinal anastomosing chain, feeding small arteries and veins in the perineurium and a relatively sparse endoneurial capillary plexus. The passage of the small vessels through the perineurium is oblique, and within the nerve funiculus the capillaries form loops (Lundborg, 1970). Human peripheral nerves can be elongated by 6 to 12 per cent without damage (Sunderland, 1968), and this remarkable degree of lengthening is made possible by the strength and elasticity of the stroma, which protects the gel-like axon (Seddon, 1975). The anatomical disposition of the blood vessels permits stretching of the nerve to occur without causing ischaemia. The longitudinal vascular chain anastomoses freely, providing for an abundant collateral supply (fig 1). Capillaries are usually permeable to solutes but in nerve funiculi diffusion occurs through the walls of venules, most if not all of which are to be found in the perineurium. There are tight junctions between the endothelial cells of the endoneurial capillaries, rendering them relatively non-permeable (Low, 1976).

\section{NERVE COMPRESSION}

The resistance of peripheral nerves to ischaemia

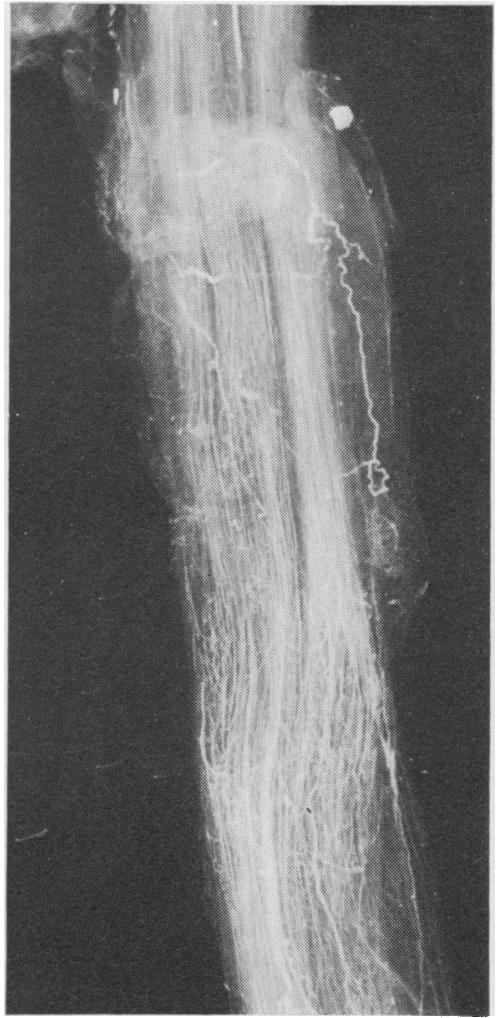

Fig. 1 Radio graph of poster- -0 ior tibial nerveo injected after? death with Mice ropaque. The longitudinally $\stackrel{2}{\mathrm{D}}$ disposed vessels are fed by epineurial arteries, some of which shom anastomosings arcades. $\times 4$

may be partly due to their structure, composed as they are of axons fed (at least partially) from the perikaryon, and able to survive independently of their satellite (Schwann) cells. As in the case of muscle, a pneumatic tourniquet can be used for upg. to two hours to produce a bloodless field during operations on nerves, without apparent ill effece (Daube and Dyck, 1975). If a limb is rendered ischaemic by an inflated cuff, motor and sensory ${ }_{\mathrm{E}}$ functions are impaired in 15 to 45 minutes. Directo pressure on a nerve has a similar effect, but Sinclair (1948) reported that anaesthesia occasionally appeared earlier than following cuff compression $\stackrel{?}{+}$ The nerve rendered ischaemic by a limb cuff foro 30 to $\mathbf{4 0}$ minutes rapidly recovers function after the circulation is restored, but, as everyone knows 
function is at first abnormal and paraesthesiae are experienced. Full restoration of function requires anything from a minute to several hours or days, according to different reports (Sunderland, 1968).

\section{ACUTE COMPRESSION}

In recent years the view that the effects of nerve compression are entirely or always mediated by ischaemia has been challenged. In his Oliver Sharpe lecture at the Royal College of Physicians, Gilliatt (1975) pointed out that acute peripheral nerve compression produces three distinct grades of injury depending on its severity and duration, namely, (1) a rapidly reversible physiological block, (2) a local demyelinating block and (3) Walleriantype degeneration. Grade 1 is known to be due to ischaemia; compressing a nerve by lying on it makes it 'go to sleep', and relief of the compression allows it to recover rapidly. Grade 2 was formerly thought to be due to ischaemia, and is caused by the more severe compression produced by an inflated cuff. Gilliatt and his colleagues showed that in baboons a standard two-hour period of compression of a limb by a sphygmomanometer cuff did not produce conduction block until pressures well above those needed to produce ischaemia were used. (Conduction block implies loss of myelin with preservation of the axon.) When the cuff pressure was $1000 \mathrm{~mm} \mathrm{Hg}$ a conduction block was regularly produced; $500 \mathrm{~mm} \mathrm{Hg}$ produced some demyelination but $250 \mathrm{~mm} \mathrm{Hg}$ produced only rapidly reversible physiological block. All three pressure levels were presumed to cause ischaemia, yet only the highest produced evidence of demyelination. The pathological change caused by the higher pressures was in the form of mechanical damage to the nerve fibres immediately deep to the edges of the cuff: nodes of Ranvier were squeezed along the nerve fibre like an intussusception, their new position being within the adjacent internode. The myelin of the compressed internodes was thinned, and after a few days during which the invaginated nodes returned to their normal position, the thinned myelin broke down leading to paranodal demyelination and remyelination (Ochoa et al, 1972). The same changes have been seen in man in a patient who was in a state of decorticate rigidity which caused compression of the median nerve for some hours before death (Neary et al, 1975).

When acute compression lasted longer than two hours, increasing numbers of nerve fibres showed Wallerian-type degeneration, a much more severe and longer lasting injury than paranodal demyelination. It was attributed to a combination of direct pressure and ischaemia. Lundborg (1970) showed in rabbits that damage to blood vessels and necrosis of muscle occurred under the cuff after four hours, and distal to the cuff after six to eight hours, supporting the contention that ischaemia participated in the pathogenesis of the lesions.

\section{CHRONIC COMPRESSION}

Gilliat and his colleagues have also found mechanical deformation of peripheral nerve fibres in chronic compression at least in one animal model (the spontaneously occurring carpal tunnel compression in elderly guinea pigs), and in human carpal tunnel and cubital tunnel syndromes affecting the median and ulnar nerves respectively. Deep to the constricting fibrous band or ligament internodes may be completely demyelinated, but proximal and distal to the lesion successive myelin sheaths are squeezed to form swellings adjacent to nodes of Ranvier, the direction of the squeeze being polarized away from the lesion in both proximal and distal directions. The swellings resemble those we have observed in myxoedema in a necropsy study (fig 2). The swellings are not associated with invagination of the node of Ranvier, but the terminal loops of myelin close to the node are detached from the axon, allowing the sheath to slip away from the node.

Sir Sydney Sunderland (1976) does not accept that the effects of chronic compression are mediated by distortion of the nerve fibres, although he grants that the role of mechanical deformation in the acute compressive lesion has been convincingly demonstrated. The most persuasive of his arguments in favour of an ischaemic pathogenesis of the chronic compressive lesion is to recall that some patients experience immediate relief of pain and recovery of function after decompression of the carpal tunnel, and this favours an improvement in the circulation rather than 'time-consuming correction of structural abnormalities in nerve fibres'. Our contribution to the debate is to show that compression alone can produce paranodal swellings, paranodal demyelination, segmental demyelination and remyelination. All these are found in myxoedema (fig 2), in which there is a great increase in intraneural mucopolysaccharide capable of compressing nerve fibres and in which ischaemia is unlikely to operate as neural blood vessels look normal by light microscopy. The issue remains sub judice.

Experience with amputation specimens teaches that neural ischaemia producing pain severe enough to warrant amputation is generally caused not by visible disease of capillaries, arterioles or venules but by atheromatous occlusion and thrombosis of proximal major arteries. There is such a good collateral circulation that small vessel disease would have to be universal to produce ischaemia. One known pathological change in capillaries that could 


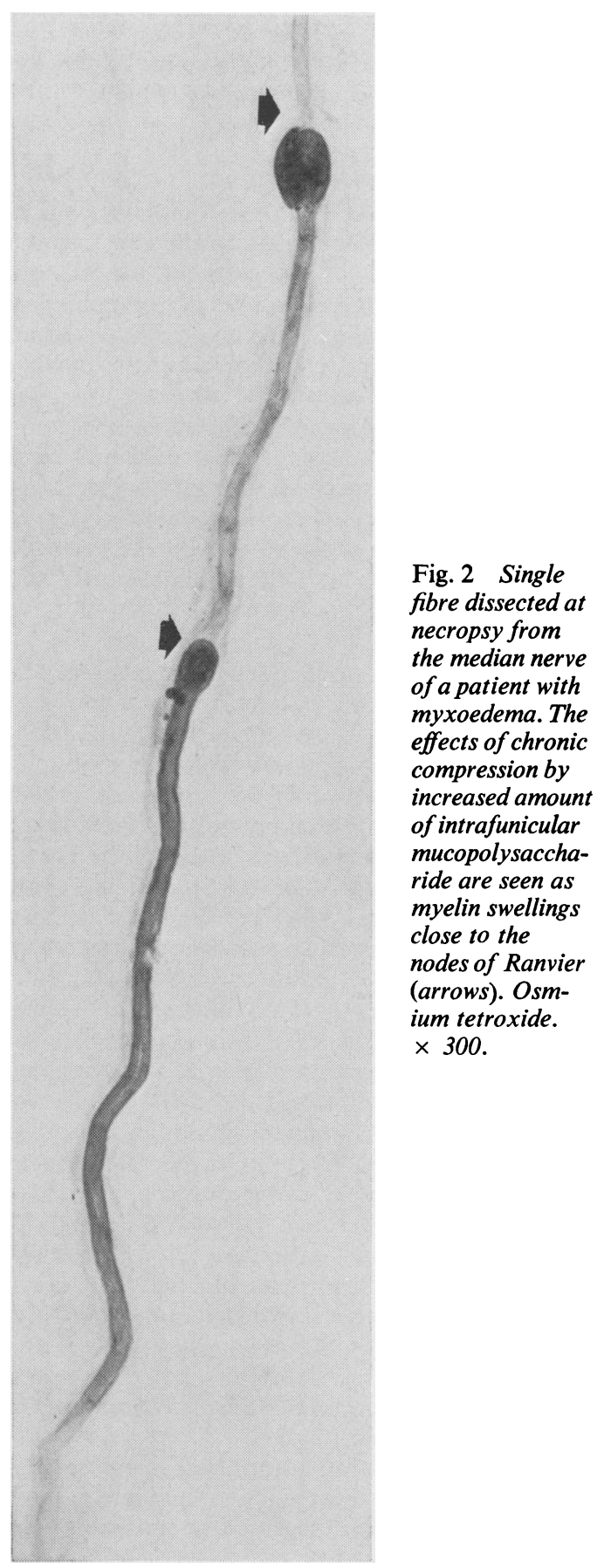

operate in this way is the basement membrane thickening of diabetic microangiopathy but it is by no means established that this change causes diabetic neuropathy (Thomas and Eliasson, 1975), let alone that it could be responsible for the amputa tion of a lower limb.

NEUROVASCULAR COMPRESSION SYNDROMES There are many chronic nerve compression an entrapment disorders in man, and uncertainty ove their pathogenesis persists. Little is known of thei histopathology, with the exception of the carpaç. tunnel syndrome. Generically they are often referred to as neurovascular compression syndromes (Robu and May, 1975) implying that both mechanical nerve compression and ischaemia play a part. In $\mathbb{P}$ review, Aguayo (1975) states that it has not been possible in experimental nerve compression clearle to separate ischaemia from mechanical factors, $s \frac{5}{8}$ that the precise pathogenesis of the nerve lesions remains a matter of conjecture. Fullerton (1963) found that patients with the carpal tunnel syndromewere more susceptible than controls to the effects of ischaemia produced by a pneumatic cuff, and that the susceptibility was related to the severity of pairf and paraethesiae. Recovery occurred rapidly afterrelease of the cuff. Two mechanisms were postulated one a rapidly reversible change in the nerve fibres: associated with ischaemic attacks and the other slowly developing structural change in the fibres resulting from pressure. The second mechanism lgas now been demonstrated in the form of paranod swellings of the nerve fibres.

The carpal tunnel is probably the commonest sitte of neurovascular compression, but there are manyo others in the upper limb: compression of the brachia plexus by a cervical rib or ligament; the ulna尺 tunnel syndrome; compression of the median nerve $\overrightarrow{6}$ in the forearm (the pronator syndrome); in the lower limb the femoral nerve may be compressed as if overlies the iliacus muscle, the saphenous nerve as if. passes through the subsartorius fascia, the sciation nerve at the inferior edge of the greater sciatic notch and the lateral peroneal nerve at it winds around the head of the fibula producing a weak ankleis (Sidey, 1969).

Neurovascular peripheral nerve compression mayo go unrecognized during life: we have noted neurogenic atrophy in the tibialis anterior muscle and losso of myelinated fibres in the lateral peroneal nerve as an incidental finding at necropsy.

CHRONIC ISCHAEMIA

The effects of known chronic ischaemia on nerves $\omega$ can be seen in limbs amputated on account of severe atherosclerotic occlusive arterial disease. In a series of 38 amputated legs examined in 1959/61, alf from middle-aged or elderly patients, we examined the sciatic nerve in 28 and found loss of myelinated fibres in five. More distally the musculocutaneousD 


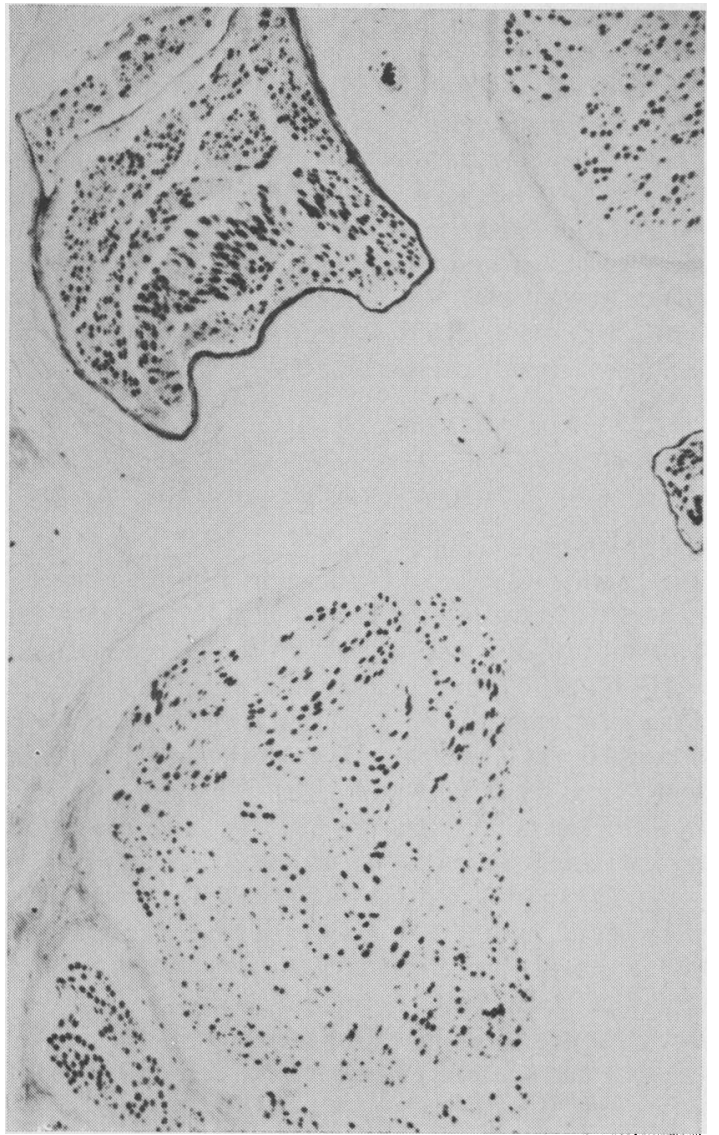

Fig. 3a

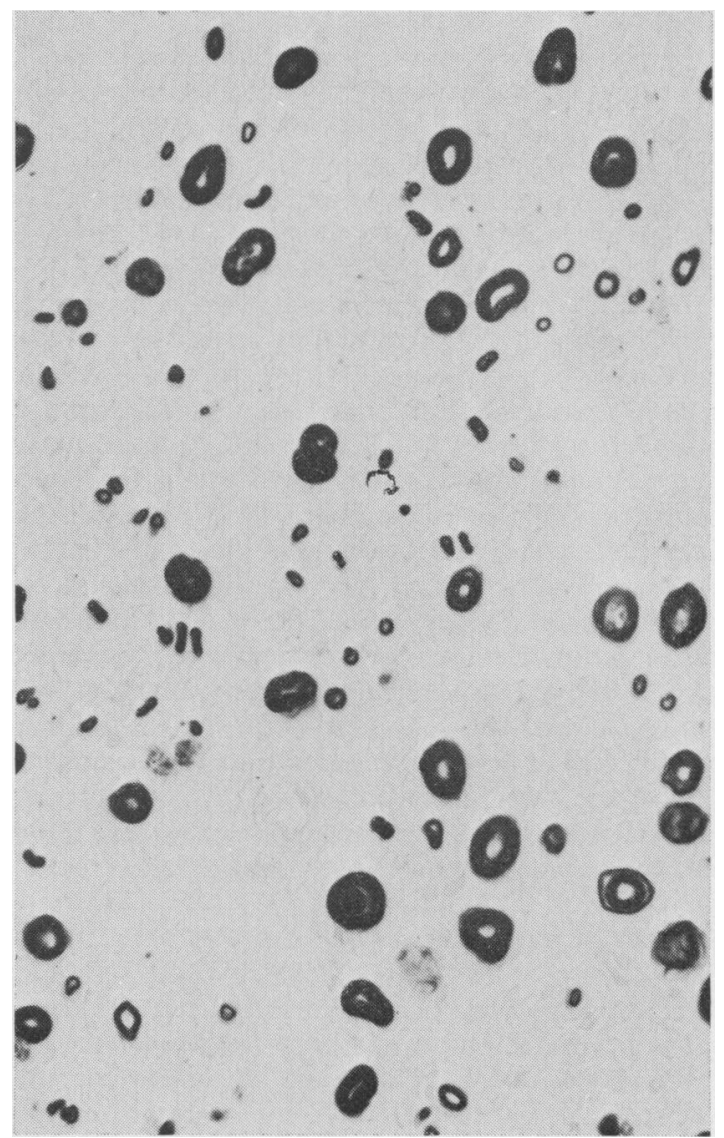

Fig. 3b

Fig. 3a Patchy loss of large and small myelinated fibres from the sciatic nerve. Above-knee amputation for athersclerotic occlusive vascular disease. Female, aged 60 years. Weigert-Pal. $\times 60$.

Fig. 3b Large and small myelinated fibres are lost in proportion. $\times 630$.

nerve showed fibre loss in 18 out of 38 specimens. Thus, when fibre loss was present, it was more severe distally. Characteristically, the loss was patchy, affecting funiculi variably (fig 3 ). Such patchy loss has also been described as characteristic of nerve compression by Aguayo (1975), providing some support for the idea that at least part of the effects of nerve compression is due to impairment of the blood supply.

\section{SELECTIVE VULNERABILITY}

Whether chronic ischaemia selectively affects nerve fibres according to their calibre has been a matter of controversy for many years. In our series of amputated limbs fibre loss appeared to affect large and small myelinated fibres proportionally, although fibre counts were not done. Selective loss of small myelinated fibres was not found, although it has been established that these fibres transmit impulses from pain receptors, and that they undergo selective degeneration in some painful neuropathies (Dyck et al, 1976), for example in amyloid neuropathy. The literature reflects as much controversy regarding selective vulnerability as it does on the comparative roles of ischaemia and mechanical deformation of nerve fibres in severe compression. Heinbecker (1929), Hershey and Wagman (1966) and Fox and Kenmore (1967) in conduction rate studies reported that ischaemia affected small fibres more than large, but Clark et al (1935) and Gasser (1942) came to the opposite conclusion. Ruskin et al (1967) maintained that the effects of ischaemia varied from subject to subject; in some there was greater susceptibility of small fibres, in others the large fibres were more affected. The differential effect on nerve fibres of different calibres was thus unpredictable, probably, they 
thought, because of the great variation in the neural capillary network between individuals. It is difficult to reconcile such conflicting results except by postulating misinterpretation of neurophysiological findings. Histological studies of the differential effect of ischaemia on myelinated and unmyelinated fibres in man are lacking (Daube and Dyck, 1975), so that, at present, a firm conclusion cannot be reached.

Do the component fibres and cells in peripheral nerves vary in their susceptibility to ischaemia? The most convincing evidence that in man Schwann cells are more sensitive than axons to ischaemia is provided by Eames and Lange (1967). Sural nerve biopsies were obtained from 32 patients immediately before amputation of a lower limb for occlusive arterial disease. The nerve showed loss of myelinated axons, and a few were undergoing the fragmentation of axon and myelin indicative of Wallerian-type degeneration. But dissection of single nerves revealed in addition extensive segmental demyelination and remyelination, showing that in the affected fibres myelin had undergone degeneration with preservation of the axon. Electron microscopy showed that unmyelinated axons were normal so that these fibres can be considered the most resistant to ischaemia.

\section{VASCULAR DISEASE AND ISCHAEMIA}

May disease of neural blood vessels be so severe as to cause nerve ischaemia? Polyarteritis nodosa may cause nerve infarction, or more frequently varying proportions of Wallerian-type degeneration and segmental demyelination and remyelination. Biopsy investigation of patients with peripheral neuropathy sometimes reveals allergic angiitis. Buerger's disease is a rare cause of lower limb ischaemia, generally over many years. Rheumatoid neuropathy occurs in two forms, one of which is ischaemic in origin and due to necrotizing arteritis (Haslock et al, 1970). In diabetic neuropathy the intraneural capillaries often show thickening of their basement membrane. Whether or not this microangiopathy results in ischaemia has not been settled (Thomas and Eliasson et al, 1975). There is a tendency to assume that thickening of capillary walls must lead to impaired diffusion, but the thickened basement membrane may in fact be more not less 'leaky' than the normal. In our experience diabetic microangiopathy is not present in young subjects in the initial weeks following the onset of diabetic neuropathy, so that an ischaemic cause for this condition is unlikely. A true ischaemic neuropathy does occur in diabetics, but is due to atherosclerotic occlusive arterial disease strictly comparable to that in non-diabetics. Eames and Lange (1967) found occlusive changes in the vasa nervorum, even thrombosis, and there is no reason to doubt that if extensive enough to outweigh the abundant collateral circulation they could be responsible fo nerve ischaemia and for pain. But it is not cleas whether small vessel occlusion on its own has eve $\overline{\underline{D}}$ been severe enough to warrant amputation. For this: to be necessary the femoral or iliac arteries or the aorta must be severely occluded. In such large arteries the lumen must be reduced to $2 \mathrm{sq} \mathrm{mm}$ oㄹ. less before blood flow is significantly impaires (Brice et al, 1964). Resistance to flow occurs largel 18 in the smallest vessels; in large ones a constrictiog induces only a local increase in the speed of flow.

\section{ARTERIAL EMBOLISM}

Arterial embolism is a rare cause of ischaemig్ neuropathy. The following case illustrates its effects

Case history

The patient was a man of 68 years. There had been $a^{\prime}$ history of recurrent abdominal pain for 12 years, but laparotomy and pyloroplasty at the commencemento of his illness had not revealed the expected duodenat ulcer. In early 1977 the peripheral pulses were described as normal. He developed melaena $\overrightarrow{{ }_{c}}$ abdominal tenderness and distension, and when seero by $\mathrm{Mr}$ Doig on 13 January 1977 there was evidence् of low small intestinal obstruction. At laparotom peritonitis and a gangrenous Meckel's diverticuluna were found, along with a short length of doubtfuifly viable small bowel. The following day femofab embolism occurred, necessitating immediate bolectomy. Embolus and clot were obtained fromp the external iliac to the superficial femoral arteriesa After this a catheter passed easily into the wholø artery, and a good back flow was obtained. Although circulation to the limb had been restored, pairp continued and on 17 January 1977 the leg was amputated 3 in above the knee, three days after the embolism.

The right leg was examined within $30 \mathrm{~min}$ of removal. The skin was normal apart from a smals area of dusky red discoloration over the lateraf. malleolus. Proximal muscles, hamstrings and quadê riceps showed large, well defined pale patcheᄍె suggesting infarction (fig 4). Below the knee, muscleg were of normal colour. The stump of the sciatio nerve, apart from surgical tension artefact, was. grossly normal, but the posterior tibial and laterah peroneal nerves, of normal whiteness proximally, gradually became soft and grey from mid calf levet distally. The distal femoral, popliteal and posteriot tibial arteries showed little atheroma, but werE occluded by dark adherent clot from the point of amputation to mid calf level. Beyond this the pos terior tibial artery was empty. Calf veins were throm? bosed but not the major veins.

The histological changes in the nerves illustrate virtually complete acute infarction. There is foca\$ 


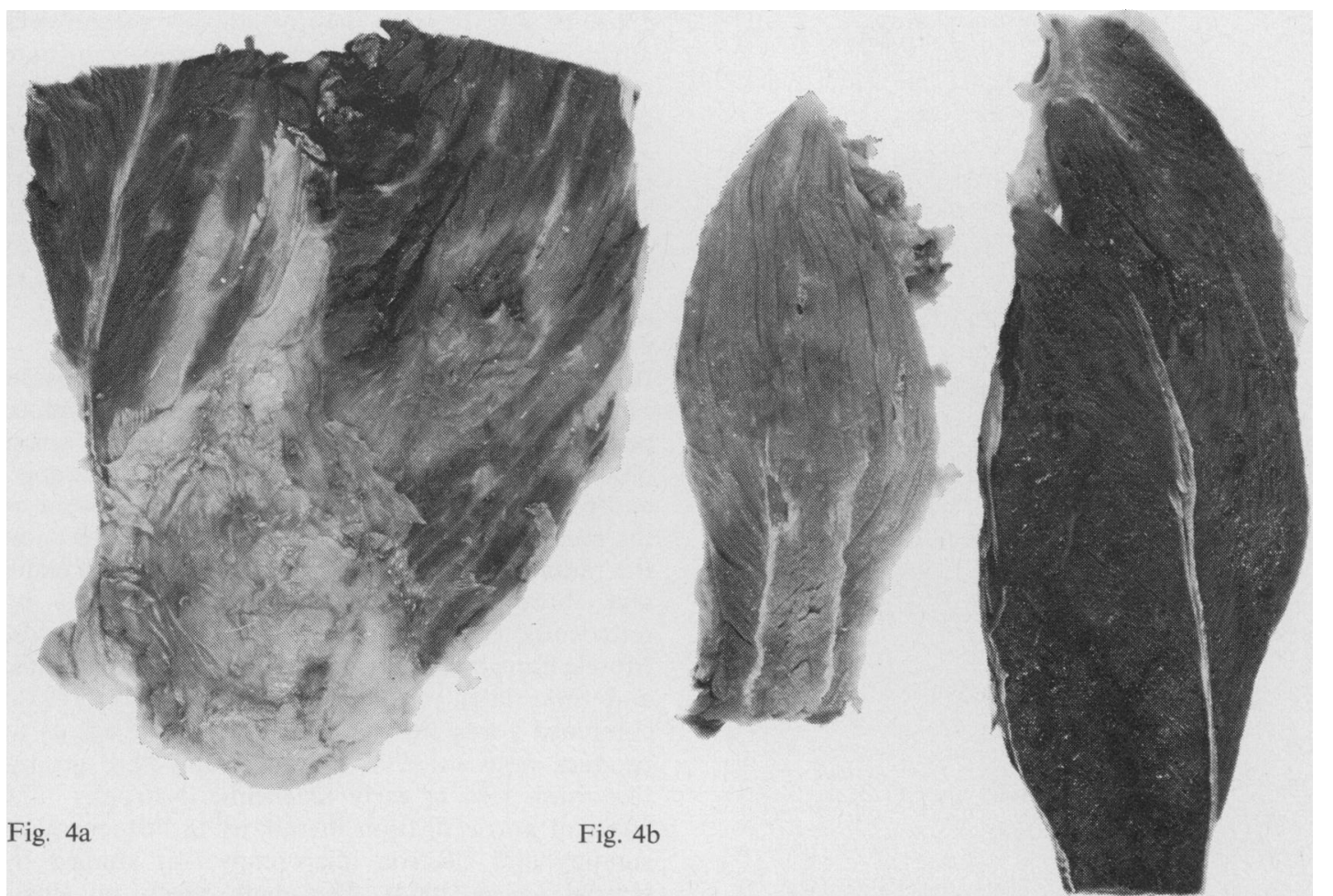

Fig. 4 Above-knee amputation for femoral embolism. (a) Lower part of quadriceps with patella, showing grey patches of infarction. (b) Two parts of the hamstrings, one infarcted, the other normal.

swelling of all myelin sheaths and of axons, and a scanty cellular reaction in the epineurium only. The nerves also show some loss of myelinated fibres, mainly of large calibre and to a greater degree in the distal nerve. This loss must have occurred well before the embolism, probably the result of aging.

Chronic infarction reduces a peripheral nerve to a thick fibrous string, and is seen most characteristically in Volkmann's ischaemic contracture (Seddon, 1975). This, involving nerve, muscle and even bone and tendon on occasion, occurs when war wounds sever an artery, or when an artery is injured alongside a fracture, or rarely as a result of embolism.

\section{Muscle}

\section{BLOOD SUPPLY}

Skeletal muscles have a rich blood supply, as would be expected in a tissue in which the blood flow is known to increase several fold within a few seconds, as a result of exercise or emotion (Dornhurst, 1963). One or more nutrient arteries enter the muscle from neighbouring major vessels, and within the muscle branch repeatedly until a capillary bed is formed (fig 5). The capillary plexus surrounds individual muscle fibres, and the capillary density is different in 'red' muscle and 'white' muscle (for an account of muscle fibre types, see Harriman, 1976). There have been few studies of capillary density in man, although capillaries are easily seen in transverse cryostat sections of muscle stained by PAS and then treated with amylase to remove glycogen. Using a similar technique in needle biopsies of the vastus lateralis, Andersen (1975) confirmed that the number of capillaries enclosing type 1 fibres which depend on oxidative metabolism was greater than around type 2 , which employ anaerobic glycolysis. Capillary density could be increased by physical training: the capillary/fibre ratio in untrained volunteers was $1 \cdot 30: 1$, and in the endurance-trained $2 \cdot 15: 1$. Figures obtained in other species are higher (mean $3 \cdot 2$ to 4.0, Plyley and Groom, 1975). The vascular supply in human muscle was studied by Blomfield (1945), who described a pattern of longitudinal anastomoses formed from one or more nutrient arteries. Muscle hypertrophy and denervation atrophy both alter the vasculature. Although muscle and peripheral nerve are similar in being provided with a rich collateral supply, capillaries are much more abundant in muscle. When blood flow is large most capillaries 


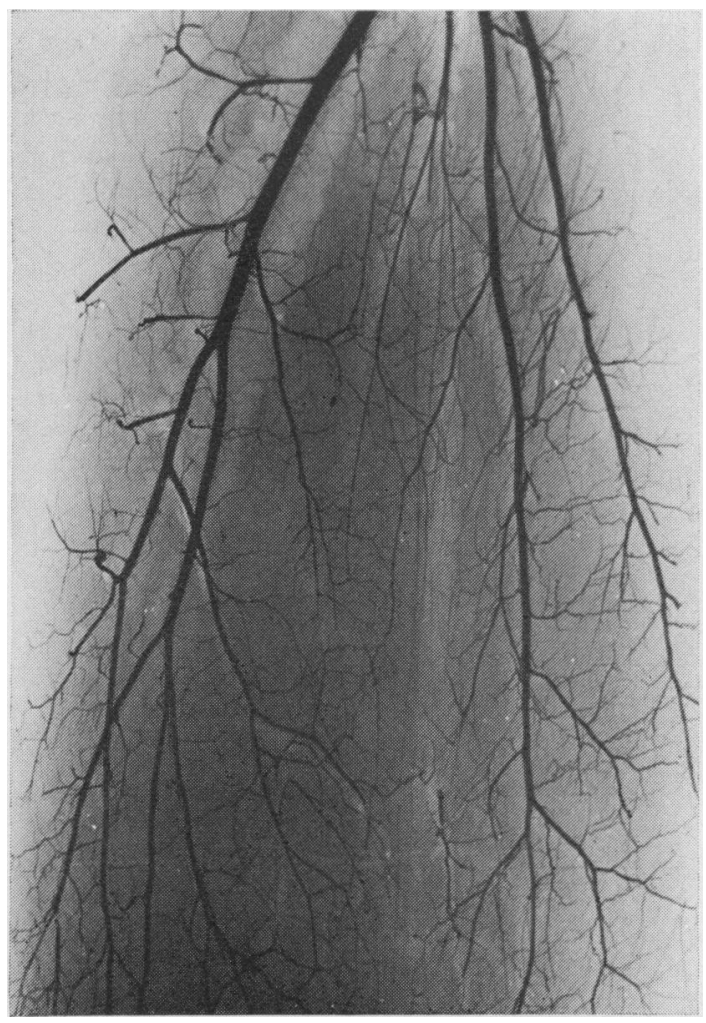

Fig. 5 Radiograph of triceps surae muscle (gastrocnemius and soleus) injected after death with Micropaque. Nutrient arteries branch dichotomously.

are presumably open, but when flow falls and the 'critical closing pressure' is reached, arteriolar elastic tension becomes zero and large numbers of vessels rapidly close (Dornhurst, 1963). In muscle, adjacent capillary endothelial cells lie in close apposition, but pinocytosis is prominent (Mair and Tomè, 1972).

\section{MYOGLOBIN}

As a carrier of oxygen in the sarcoplasm myoglobin is of great importance in some mammals (Biörk, 1949). It is responsible for the red colour of muscle, the intensity of the colour being enhanced by the opaque lipid content of the same fibres. Porpoises and other diving mammals have so much that their muscles are dark, almost black. Most of it is to be found in 'red' (type 1) muscle fibres, within which it releases oxygen for oxidative metabolism. 'Red' muscle not only possesses a more dense capillary network than 'white', but has a higher resting blood supply and a significantly greater resting oxygen consumption. In man, the myoglobin oxygen stores are of relatively little importance; they contribute up to 10 per cent of the energy consumed during 20 minutes' ischaemia at rest, but in maximal muscle activity when the metabolic rate is increased $a^{?}$ hundred fold this contribution is negligible (Edwards? 1976). The metabolic rate of resting human muscle $\frac{\bar{c}}{\bar{c}}$ is very low (Harris et al, 1975) so that the effect of ischaemia can be expected to be enhanced by muscle activity.

EXPERIMENTAL ISCHAEMIA

It is not easy to produce ischaemic myopathy inpure form; such procedures as aortic ligation reduces perfusion pressure in hind limbs but do not arrest blood flow completely because of collateral supplyo and peripheral nerves are as liable to be affected asw the muscles, with consequent denervation effects on the muscle. Hathaway et al (1970) produced acuteand chronic ischaemic myopathy in rabbits by microembolization of the hind legs with glass beads? Muscle fibres were rendered necrotic in small groups,, and these later regenerated. Mendell et al (1971) combined aortic ligation with vasospastic agents to produce necrosis of single fibres and fibre groups like those seen in early Duchenne dystrophy. The effect of aortic ligation in rats using histochemicalo staining and electron microscopy was studied \$y. Karpati et al (1974). The acute effects on singlep fibres and groups consisted of clumping of NAF reaction product, loss of phosphorlyase activity ang of glycogen. The ATPase reactions were not affected until phagocytosis of the necrotic fibres began. Later $\mathbb{Q}$ clusters of up to 200 fibres were seen undergoing necrosis and phagocytosis. The important observa $\frac{0}{3}$ tion was made that the lesions were much moresevere in the red solei than in the predominantlyo white gastrocnemii, ie, that oxidative fibres were more susceptible than anaerobic glycolytic ones? When one limb was denervated, or rendered inactive by Achilles tenotomy or skeletal fixation the lesions. did not develop in that limb, but were more severe in the opposite limb which these procedures had rendered hyperactive. Again, red muscle was more extensively affected. Regeneration was conspicuousp at later stages, and virtually complete by three months after ligation. The lesions were not followed by fibrosis showing that muscle has considerable regenerative capacity when conditions are suitable. ㅇ

Probably the most severe experimental ischaemie lesions have been produced by Boysen and Enge $F$ (1975), who embolized the femoral artery of rabbito with dextran particles. With a massive dose, the tibialis anterior muscle showed virtually totaf necrosis and an inflammatory reaction; after six weeks there was marked variation in fibre calibre with smaller fibres at the periphery, a moderate? 
increase of central nuclei and moderate endomysial fibrosis. Other workers have used human fragmented atheromatous plaques as experimental emboli, with surprisingly little effect on muscle but providing a useful model for the study of arterial thrombi (Warren and Lytton, 1976).

\section{ISCHAEMIC MYOPATHY IN MAN}

The reaction of human skeletal muscle to ischaemia is not known in the same detail as in experimental animals, for studies have been few. The literature was reviewed by Karpati et al (1974). Fragmentation of necrotic muscle fibres into discs, foci of necrosis and infarcts have been recognized. Boehme et al (1966) examined muscles from 21 amputated limbs; there was little necrosis but much evidence of denervation atrophy in the form of atrophy of large groups of muscle fibres or of scattered single fibres. Our experience has been different. In 38 amputated lower limbs, there was evidence of neurogenic atrophy in distal muscle, the peroneus brevis, in seven, cachectic atrophy (Harriman, 1976) in seven, necrosis and regeneration in five, muscle fibre vacuolation in two, and pronounced haemosiderosis in one. Necrosis and regeneration and muscle fibre vacuolation seemed to be a direct effect of ischaemia rather than of ischaemic neuropathy, as they occurred in fibres of normal calibre. None of these specimens, however, were examined by cryostat histochemical techniques which are more sensitive indicators of histopathology than paraffin sections can be.

Three recently examined limbs, two legs amputated on account of occlusive vascular disease and one following arterial embolism (page 98), have been informative. In all three there were infarcts in proximal muscles (fig 4). Other muscles, normal in colour and consistency, showed scattered 'ischaemic' muscle fibres (fig 6). These were swollen and rounded, reacted with NADH, gave no reaction for phosphorylase, and contained no glycogen, but the reactions for myofibrillar ATPase persisted and they often contained an excess of triglyceride droplets (Harriman and Reed, 1972). Ischaemic fibres were so called as their structural abnormalities seemed to be caused specifically by anoxic ischaemia. The fibres affected were more often type 1 . They have not been reported in endocrine, inflammatory (immunological or infective) or genetically determined myopathies.

\section{SYNDROMES INVOLVING MUSCLE ISCHAEMIA} Volkmann's ischaemic contracture is the best known but is not common (see page 99). The muscular affection usually includes haemorrhage, and this gave rise to the original belief that the important vascular injury or occlusion was venous. The con-

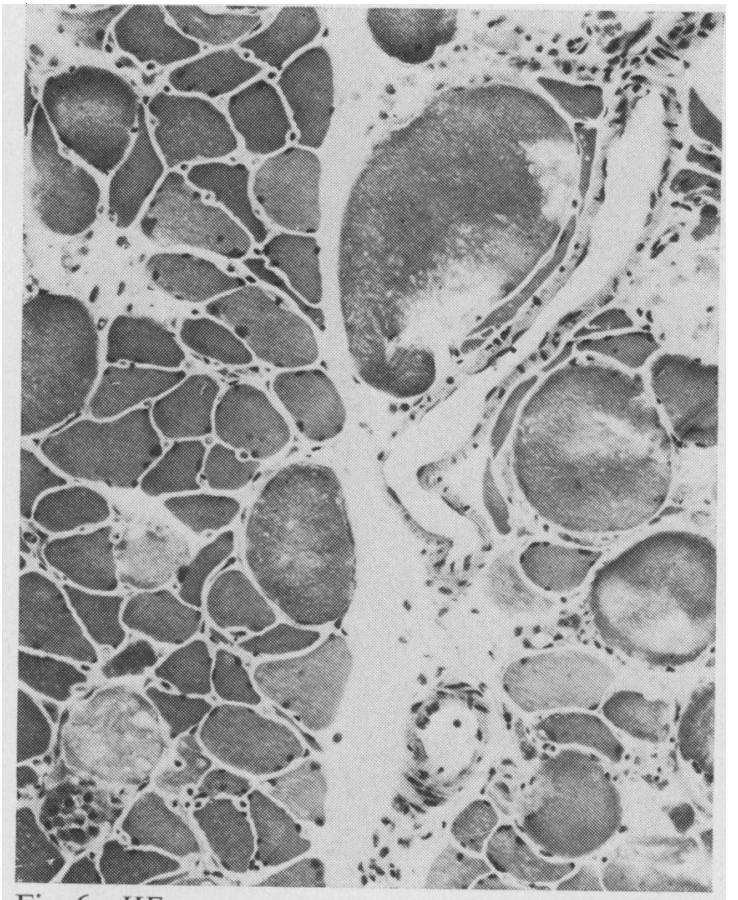

Fig. 6a $H E$

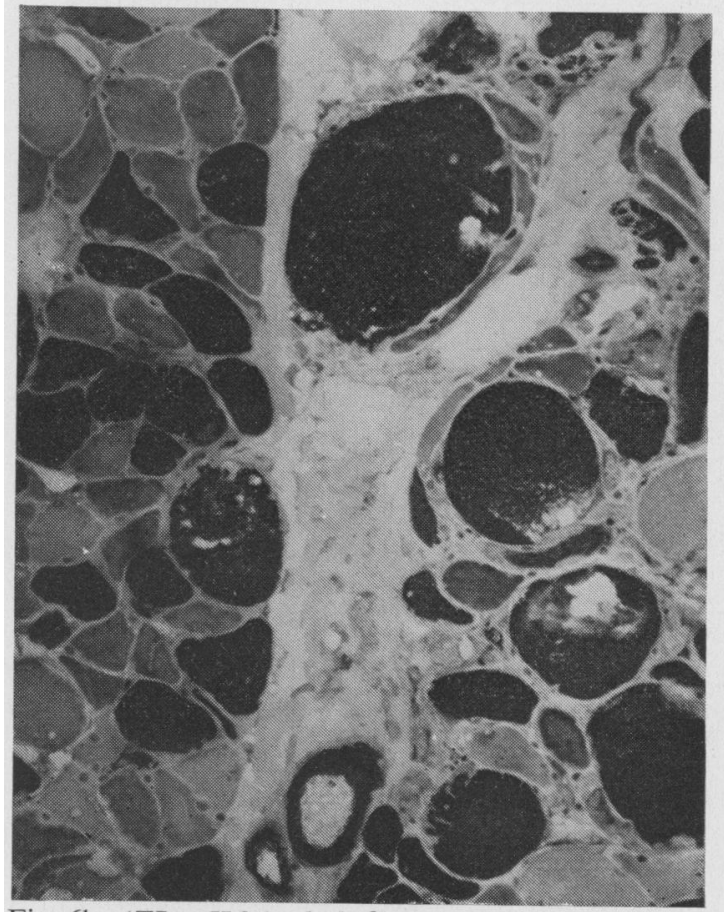

Fig. 6b ATPa pH 9.5; dark fibres type 2.

Fig. 6 Serial cryostat sections to show 'ischaemic' fibres, usually swollen, vacuolated or blotchy, mainly in fascicle to $R$. 


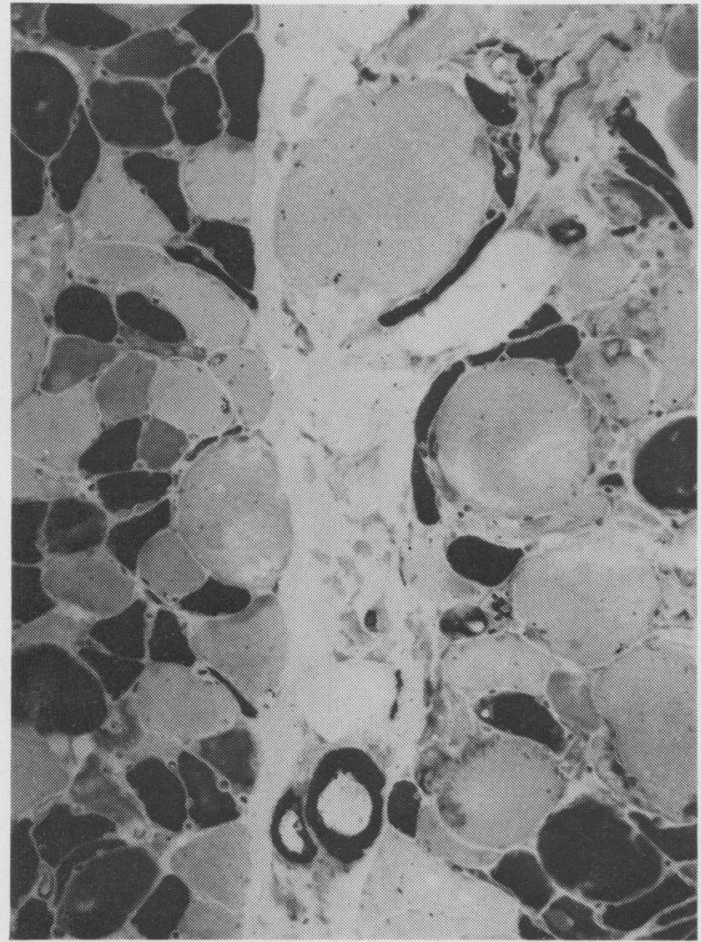

Fig. 6c ATPase $p H 4 \cdot 2$, dark fibres type 1;

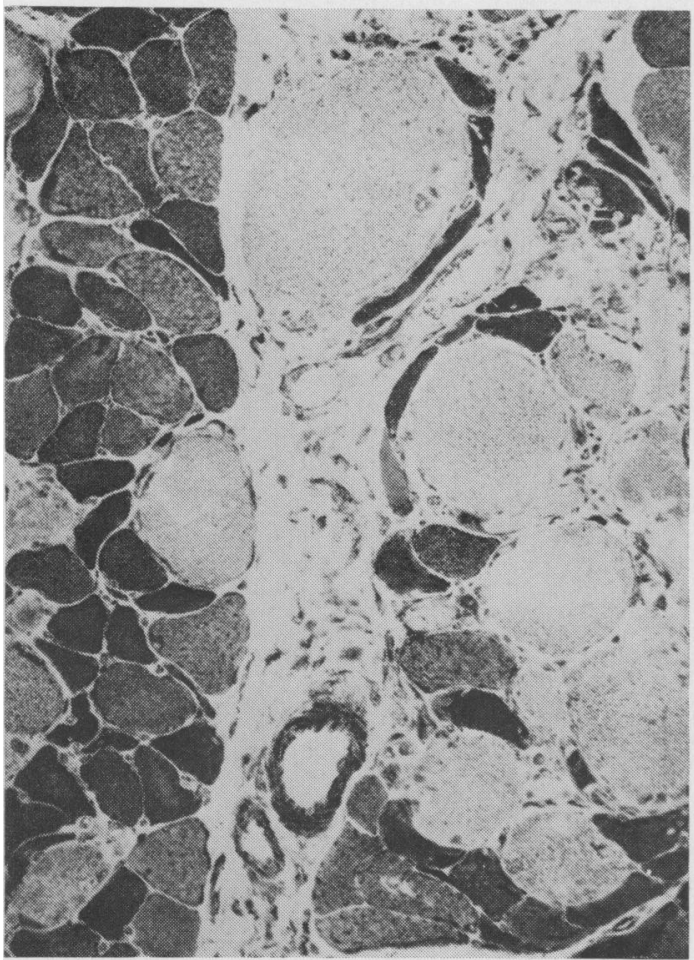

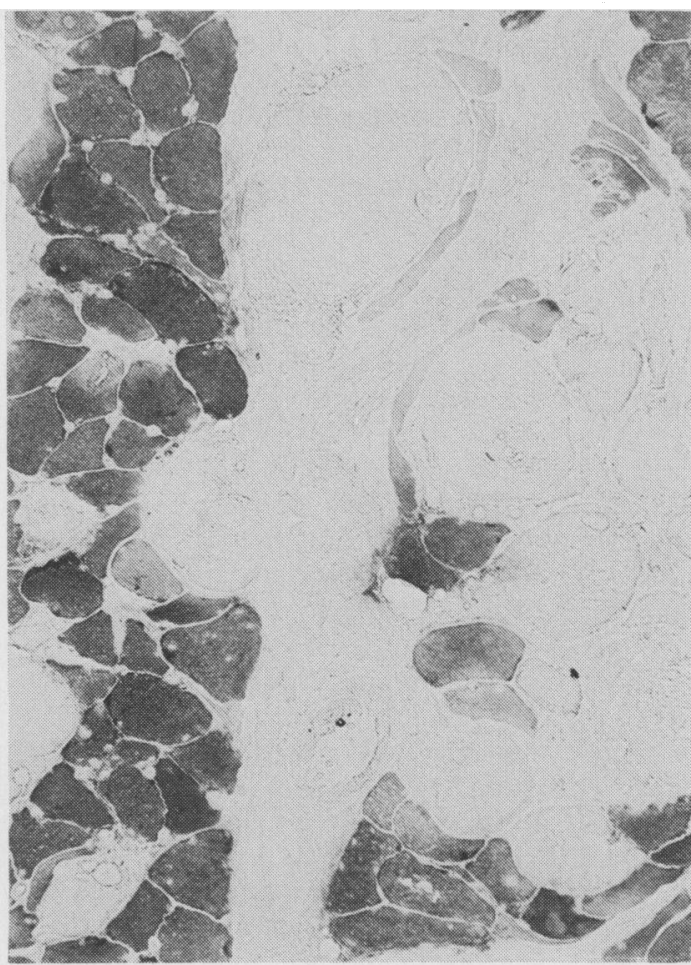

Fig. 6e phosphorylase;

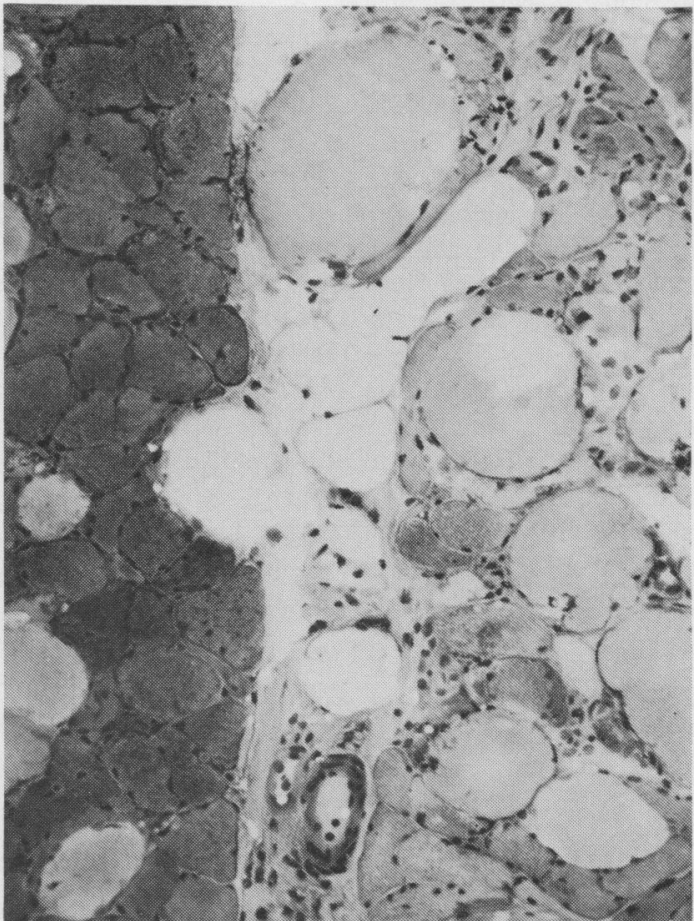

Fig. 6f PAS. All $\times 150$

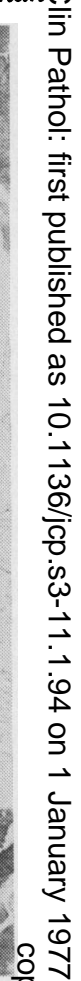
흘.

o

ฉิ

$\stackrel{\mathbb{2}}{2}$

옥

吾

응

응

을.

ํํำ 욱

을. N

กิ

స్ట

운

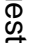

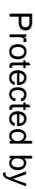

Fig. 6d NADH; 
sensus among experienced surgeons is that arteries are damaged, producing infarction of muscle as well as of other tissues. In the course of time the infarct is largely replaced by fibrous tissue, leading to contracture unless appropriate surgery is undertaken.

Acute necrosis of muscle can lead to myoglobinuria, electrolyte disturbance and acute renal failure. The necrosis may be partly of ischaemic origin in 'crush anuria', well known during the second world war, when extensive damage to muscle and its blood supply occurred in crush injuries (Bywaters et al, 1941). Probably the leading cause of myoglobinuria in the young adult population is acute exertional rhabdomyolysis. This syndrome arises in healthy individuals who have performed an excessive amount of exercise to which they are unaccustomed, particularly repetitive physical training (Geller, 1973). The cause of the condition is not known but can be surmised to be due to oedema of muscle resulting from an excess of anaerobically produced metabolites; the muscle swelling embarrasses the circulation particularly in muscles confined by bones and strong fascial septa. This mechanism presumably operates in the compartment syndromes, of which the most frequent is the anterior tibial compartment syndrome (Bradley, 1973). Damage to the muscle varies from a few foci of necrosis and regeneration to large areas of infarction leading to fibrosis and a dropped foot. At operation in the acute phase mere incision of the anterior tibial fascia relieves the patient's pain. Other muscle compartments are much less frequently affected, for example, the deep posterior compartment of the leg or the volar compartment of the forearm (Matsen and Clawson, 1975). Compartment muscles are more susceptible than others to ischaemia: infarction of the anterior tibial muscle has been caused by catheterization of the femoral artery impairing the circulation for only two and a half hours (Rosengart et al, 1976).

Just as the relative importance of compression and vascular insufficiency is not clear in the neurovascular syndromes (page 96), the same may be said of the much rarer examples of muscle compression. Prolonged immobility on a hard surface is liable to cause muscle necrosis (Clark and Sumerling, 1966; Penn et al, 1972). We have seen a biopsy of gastrocnemius from an adult patient who lay in a coma for many hours from carbon monoxide anoxia. Consciousness was restored, but the patient suffered from painfully tender calf muscles. The biopsy made some months later showed a picture not unlike polymyositis, with continuing necrosis, regeneration, small infiltrates of inflammatory cells and fibre vacuolation. A feature distinguishing it from myositis was the presence of scattered haemosiderocytes.

\section{Summary}

Although skeletal muscle and peripheral nerves are both resistant to ischaemia there are nevertheless many syndromes in which they are affected, either separately or together. It is frequently difficult to distinguish the effects of arterial ischaemia from those of compression, which may operate through vascular occlusion, or, in the case of peripheral nerve, by mechanical deformation of nerve fibres. A great deal has been learnt from experimental models, but not all of it is applicable to the complexity of human neuromuscular ischaemia which requires further study.

\section{References}

Adams, R. D. (1975). Diseases of Muscle: A study in Patho$\log y$. 3rd edition. Harper and Row, Hagerstown, London.

Aguayo, A. J. (1975). Neuropathy due to compression and entrapment. In Peripheral Neuropathy, edited by P. J. Dyck, P. K. Thomas, and E. H. Lambert, ch. 34. Saunders, Philadelphia.

Andersen, P. (1975). Capillary density in skeletal muscle of man. Acta Physiologica Scandinavica, 95, 203-205.

Biörk, G. (1949). On myoglobin and its occurrence in man. Acta Medica Scandinavica, Suppl. 226.

Blomfield, L. B. (1945). Intramuscular vascular patterns in man. Proceedings of the Royal Society of Medicine, 38, 617-618.

Boehme, D., Themann, H., and Gold, J. (1966). Structural and ultrastructural changes in striated human muscle caused by chronic ischaemia. American Journal of Patho$\log y, 49,569-591$.

Boysen, G., and Engel, A. G. (1975). Effects of microembolization on the skeletal muscle blood flow. Acta Neurologica Scandinavica, 52, 71-80.

Bradley, E. L. (1973). The anterior tibial compartment syndrome. Surgery, Gynecology and Obstetrics, 136, 289-297.

Brice, J. G., Dowsett, D. J., and Lowe, R. D. (1964). Haemodynamic effects of carotid artery stenosis. British Medical Journal, 2, 1363-1366.

Bywaters, E. G. L., Delory, G. E., Remington, C., and Smiles, J. (1941). Myohaemoglobin in the urine of air-raid casualties with crushing injury. Biochemical Journal, 35, 1164-1168.

Clark, D., Hughes, J., and Gasser, H. S. (1935). Afferent function in the group of nerve fibers of slowest conduction velocity. American Journal of Physiology, 114, 69-76.

Clark, J. G., and Sumerling, M. D. (1966). Muscle necrosis and calcification in acute renal failure due to barbiturate intoxication. British Medical Journal, 2, 214-215.

Daube, J. R., and Dyck, P. J. (1975). Neuropathy due to peripheral vascular diseases. In Peripheral Neuropathy, edited by P. J. Dyck, P. K. Thomas, and E. H. Lambert, ch. 35. Saunders, Philadelphia.

Dornhurst, A. C. (1963). Clinical implications of recent peripheral vascular studies. British Medical Bulletin, 19, 161-162.

Dyck, P. J., Lambert, E. H., and O'Brien, P. C. (1976). Pain in peripheral neuropathy related to rate and kind of fibre degeneration. Neurology (Minneapolis), 26, 466-471.

Eames, R. A., and Lange, L. S. (1967). Clinical and pathological study of ischaemic neuropathy. Journal of Neurology, Neurosurgery and Psychiatry, 30, 215-226. 
Edwards, R. H. T. (1976). Energy metabolism in normal and dystrophic human muscle. In Proceedings of the Muscular Dystrophy Association of America Inc. Conference, Durango, Colorado. Excerpta Medica International Congress Series, Amsterdam. (To be published.)

Fox, J. L., and Kenmore, P. I. (1967). The effect of ischemia on nerve conduction. Experimental Neurology, 17, 403-419.

Fullerton, P. M. (1963). The effect of ischaemia on nerve conduction in the carpal tunnel syndrome. Journal of Neurology, Neurosurgery and Psychiatry, 26, 385-397.

Gasser, H. S. (1942). Pain producing impulses in peripheral nerves. Association for Research in Nervous and Mental Disease, Proceedings, 23, 44-62.

Geller, S. A. (1973). Extreme exertion rhabdomyolysis. A histopathologic study of 31 cases. Human Pathology, 4, 241-250.

Gilliatt, R. W. (1975). Peripheral nerve compression and entrapment. (The Oliver Sharpey Lecture). In Proceedings of the 11th Symposium on Advanced Medicine, Royal College of Physicians of London, February, 1975, edited by A. F. Lant, pp. 144-163. Pitman, London.

Harriman, D. G. F. (1976). Diseases of muscle. In Greenfield's Neuropathology, 3rd edn, edited by W. Blackwood and J. A. N. Corsellis, ch. 19. Arnold, London.

Harriman, D. G. F., and Reed, R. (1972). The incidence of lipid droplets in human skeletal muscle in neuromuscular disorders: a histochemical, electron-microscopic and freeze-etch study. Journal of Pathology, 106, 1-24.

Harris, R. C., Hultman, E., Kaijser, L., and Nordesjo, L. O. (1975). The effect of circulatory occlusion on isometric exercise capacity and energy metabolism of the quadriceps muscle in man. Scandinavian Journal of Clinical and Laboratory Investigation, 35, 87-95.

Haslock, D. I., Wright, V., and Harriman, D. G. F. (1970). Neuromuscular disorders in rheumatoid arthritis: a motor-point muscle biopsy study. Quarterly Journal of Medicine, 34, 335-358.

Hathaway, P. W., Engel, W. K., and Zellweger, H. (1970). Experimental myopathy after microarterial embolization: Comparison with childhood X-linked pseudohypertrophic muscular dystrophy. Archives of Neurology, 22, 365-378.

Heinbecker, P. (1929). Effect of anoxemia, carbon dioxide and lactic acid on electrical phenomena of myelinated fibers of the peripheral nervous system. American Journal of Physiology, 89, 58-83.

Hershey, W. N., and Wagman, I. H. (1966). Effects of ischemia on range of conduction velocities and on facilitation in human motor nerves. Transactions of the American Neurological Association, 91, 246-248.

Karpati, G., Carpenter, S., Melmed, C., and Eisen, A. A. (1974). Experimental schemic myopathy. Journal of the Neurological Sciences, 23, 129-161.

Low, F. N. (1976). The perineurium and connective tissue of peripheral nerve. In The Peripheral Nerve, edited by D. N. Landon, ch. 3. Chapman and Hall, London.

Lundborg, G. (1970). Ischemic nerve injury. Scandinavian Journal of Plastic and Reconstructive Surgery, Suppl. 6.

Mair, W. G. P., and Tomè, F. M. S. (1972). Atlas of the Ultrastructure of Diseased Human Muscle, Churchill Livingstone, Edinburgh and London.
Matsen, F. A., III and Clawson, D. K. (1975). The deep posterior compartmental syndrome of the leg. Journal of Bone and Joint Surgery, 57, A 34-39.

Mendell, J. R., Engel, W. K., and Derrer, E. C. (1971). Duchenne muscular dystrophy; functional ischemia reproduces its characteristic lesions. Science, 172, 11431145.

Neary, D., Ochoa, J., and Gilliatt, R. W. (1975). Sub-clinical entrapment neuropathy in man. Journal of the Neurological Sciences, 24, 283-298.

Ochoa, J., Fowler, T. J., and Gilliatt, R.W.(1972). Anatomical changes in Peripheral nerves compressed by a pneumatic tourniquet. Journal of Anatomy, 113, 433-455.

Penn, A. S., Rowland, L. P., and Fraser, D. W. (1972). Drugs coma and myoglobinuria. Archives of Neurology, 26, 336-343.

Plyley, M. J., and Groom, A. C. (1975). Geometrical distribution of capillaries in mammalian striated muscle. American Journal of Physiology, 228, 1376-1383.

Rob, C., and May, A. G. (1975). Neurovascular compression syndromes. Advances in Surgery, 9, 211-234.

Rosengart, R., Nelson, R. J., and Emmanoulides, G. C. (1976). Anterior tibial compartment Syndrome in a child: an unusual complication of cardiac catheterization. Pediatrics, 58, 456-458.

Ruskin, A. P., Tanyag-Jocson, A., and Rogoff, J. B. (1967). Effect of ischemia on conduction of nerve fibers of varying diameters. Archives of Physical Medicine and Rehabilitation, 48, 304-310.

Seddon, H. J. (1975). Surgical Disorders of the Peripheral Nerves, 2nd edn. Churchill Livingstone, Edinburgh, London and New York.

Sidey, J. D. (1969). Weak ankles: a study of commog peroneal entrapment neuropathy. British Medical Journat 3, 623-626.

Sinclair, D. C. (1948). Observations on sensory paralys produced by compression of a human limb. Journal of Neurophysiology, 11, 75-92.

Sunderland, S. (1945a). Blood supply of the nerves of the upper limb in man. Archives of Neurology and Psychiatry, 53, 91-115.

Sunderland, S. (1945b). Blood supply of peripheral nerves: practical considerations. Archives of Neurology and Psychiatry, 54, 280-282.

Sunderland, S. (1945c). Blood supply of the sciatic nerve and its popliteal divisions in man. Archives of Neurology and Psychiatry, 54, 283-289.

Sunderland, S. (1968). Nerves and Nerve Injuries, ch. 6. Livingstone, Edinburgh.

Sunderland, S. (1976). The nerve lesion in the carpal tunnel syndrome. Journal of Neurology, Neurosurgery and Psychiatry, 39, 615-626.

Thomas, P. K., and Eliasson, S. G. (1975). Diabetic neuropathy. In Peripheral Neuropathy, edited by P. J. Dyck, P. K. Thomas, and E. H. Lambert, ch. 47. Saunders. Philadelphia.

Warren, B. A., and Lytton, D. G. (1976). The effects and $\bar{N}$ morphology of atheroembolism in limb arteries: an $\mathscr{\Omega}$ experimental study. Pathology, 8, 231-245. 